\title{
The endoplasmic reticulum in plant immunity and cell death
}

\section{Ruth Eichmann and Patrick Schäfer*}

The School of Life Sciences, University of Warwick, Coventry, UK

\section{Edited by:}

Jacqueline Monaghan, The Sainsbury Laboratory, UK

\section{Reviewed by:}

Yusuke Saijo, Max Planck Institute for Plant Breeding Research, Germany Frederikke Gro Malinovsky, The

Sainsbury Laboratory, UK

\section{*Correspondence:}

Patrick Schäfer, The School of Life

Sciences, University of Warwick,

Gibbet Hill Campus, Coventry

CV4 TAL, UK.

e-mail: p.schafer@warwick.ac.uk
The endoplasmic reticulum (ER) is a highly dynamic organelle in eukaryotic cells and a major production site of proteins destined for vacuoles, the plasma membrane, or apoplast in plants. At the ER, these secreted proteins undergo multiple processing steps, which are supervised and conducted by the ER quality control system. Notably, processing of secreted proteins can considerably elevate under stress conditions and exceed ER folding capacities. The resulting accumulation of unfolded proteins is defined as ER stress. The efficiency of cells to re-establish proper ER function is crucial for stress adaptation. Besides delivering proteins directly antagonizing and resolving stress conditions, the ER monitors synthesis of immune receptors. This indicates the significance of the ER for the establishment and function of the plant immune system. Recent studies point out the fragility of the entire system and highlight the ER as initiator of programed cell death (PCD) in plants as was reported for vertebrates. This review summarizes current knowledge on the impact of the ER on immune and PCD signaling. Understanding the integration of stress signals by the ER bears a considerable potential to optimize development and to enhance stress resistance of plants.

Keywords: programed cell death, plant immunity, unfolded protein response, stress, endoplasmic reticulum quality control

\section{INTRODUCTION}

The endoplasmic reticulum (ER) is an organelle with important functions in eukaryotic cells. It connects to other cellular compartments [e.g., nucleus, Golgi apparatus, mitochondria, peroxisomes, plasma membrane (PM)] and, as one of the largest $\mathrm{Ca}^{2+}$ stores, participates in intracellular $\mathrm{Ca}^{2+}$ signaling. It is further involved in lipid and hormone biosynthesis (Staehelin, 1997; Sparkes et al., 2009; Lynes and Simmen, 2011). Importantly, the ER quality control (ER-QC) system mediates and monitors the processing and folding of secretory proteins destined for transport to the PM, vacuole, or apoplast, identifies misfolded proteins and transfers them to the ER-associated degradation (ERAD) machinery (Vitale and Boston, 2008; Liu and Howell, 2010; Hüttner and Strasser, 2012). Among the proteins processed by the plant's ER-QC are important PM-resident proteins involved in adaptation to environmental stress, e.g., hormone or immune receptors (Saijo, 2010). ER integrity is central to proper function of cells and whole organisms. Especially under stress conditions, any impairment of ER function can result in disturbed plant development and plant immunity (Wang et al., 2005; Vitale and Boston, 2008; Saijo, 2010).

\section{REGULATION OF ER INTEGRITY AND ER STRESS SIGNALING IN EUKARYOTES}

Protein folding demand and capacities in the ER are usually in equilibrium. However, responses to environmental stresses create an increased requirement for secreted proteins. If this demand exceeds the ER-QC working capacity, unfolded proteins accumulate in the ER, which the cell senses as ER stress. Prolonged ER stress impairs ER function and thus threatens cellular integrity. Chemicals, such as the $N$-glycosylation inhibitor tunicamycin (TM) or the reducing agent dithiothreitol (DTT), which inhibits the formation of disulfide bonds, are widely used to induce and examine ER stress (Martínez and Chrispeels, 2003; Kamauchi et al., 2005; Vitale and Boston, 2008; Liu and Howell, 2010).

In animals, mainly three ER membrane proteins constitute the cell's ER stress surveillance system: the type I transmembrane protein kinase/endoribonuclease inositol-requiring enzyme 1 (IRE1 $\alpha$ and $\beta$ ), the type I transmembrane protein kinase RNAlike ER kinase (PERK), and the type II transmembrane basic leucine-zipper (bZIP) domain-containing activating transcription factor 6 (ATF6). In yeast cells, IRE1 is the only ER stress sensor (Mori, 2009). Under non-stressed conditions, luminal parts of these ER stress sensors bind to luminal binding proteins (BiPs), which keeps the sensors in an inactive state. If unfolded proteins accumulate, BiPs disconnect from ER stress sensors to mediate processing of unfolded proteins. Once liberated, ER stress sensors initiate different adaptive signaling cascades defined as unfolded protein response (UPR) to re-establish proper ER function. The UPR enhances the synthesis of antioxidants and ER-QC members, attenuates translation, suppresses expression of secretory genes, and elevates ERAD of unfolded proteins (Schröder, 2006, 2008; Liu and Howell, 2010; Hetz, 2012; Higa and Chevet, 2012; Jäger et al., 2012). Figure 1A summarizes processes involved in UPR activation by the three ER stress sensors in animals. BiP release allows ATF6 translocation to the Golgi apparatus, where its cytosolic part (cATF6), a functional bZIP transcription factor, is cleaved off by serine proteases S1P and S2P, a process called regulated intramembrane proteolysis (RIP). cATF6 then enters the nucleus and promotes transcription of UPR genes and the bZIP transcription factor XBP1 (Yoshida et al., 2001). Upon BiP release, IRE1 oligomerizes and activates its endoribonuclease domain, leading 


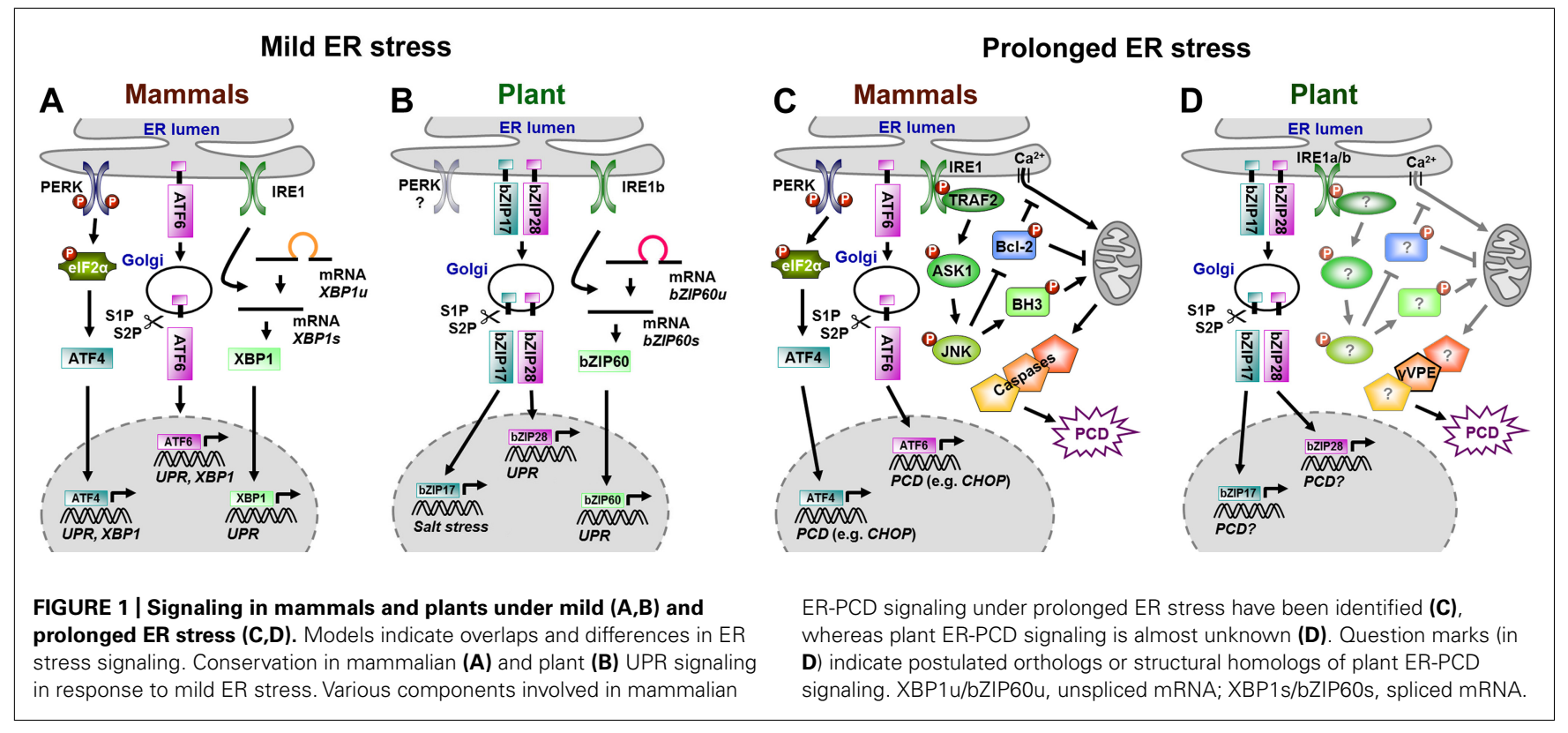

to the unconventional splicing of a 26 nucleotide intron out of $X B P 1$ or its yeast counterpart $H A C 1$, which allows the resulting proteins to enter the nucleus (Mori, 2009; Walter and Ron, 2011; Hetz, 2012). Phosphorylation by the PERK kinase activates the eukaryotic translation initiation factor eIF2 $\alpha$, which attenuates translation but selectively promotes the translation of the transcription factor ATF4 (Harding et al., 2000). Eventually, ATF4, ATF6, and XBP1 (HAC1) elevate transcription of UPR genes (Mori, 2009; Walter and Ron, 2011; Hetz, 2012).

In plants, the ER-QC and ER stress responses are apparently conserved as suggested by sequence homologies found in Arabidopsis for members of the ER translocon and oligosaccharyltransferase complexes as well as for UPR and ERAD components (Liu and Howell, 2010). Further, transcripts of genes encoding proteins of the ER-QC machinery [e.g., chaperones BiPs, CALRETICULINs (CRTs), CALNEXINs (CNXs) or PROTEIN DISULFIDE ISOMERASEs (PDIs)], or the ERAD pathway are induced by ER stress (Jelitto-Van Dooren et al., 1999; LeborgneCastel et al., 1999; Koizumi et al., 2001; Martínez and Chrispeels, 2003; Kamauchi et al., 2005; Lu and Christopher, 2008; Su et al., 2011; Hüttner and Strasser, 2012). Putative plant ER stress sensors and signaling components have been identified (Figure 1B), however, except for IRE, respective plant proteins do not show sequence but structural or functional homology (Koizumi et al., 2001; Liu and Howell, 2010). Arabidopsis possesses at least two IRE1-like proteins, while only one homolog is present in rice (Oryza sativa). AtIRE1a, AtIRE1b, and OsIRE1 harbor all structural features of yeast and mammalian IRE1. AtIRE1a and OsIRE1 are capable of autotransphosphorylation, and the putative ER stress sensor domain of AtIRE1a, AtIRE1b, and OsIRE1 can functionally replace that of yeast IRE1 (Koizumi et al., 2001; Noh et al., 2002; Okushima etal., 2002). There are at least three ER-resident transmembrane bZIP transcription factors in Arabidopsis, which are involved in ER stress responses, AtbZIP17, AtbZIP28, and AtbZIP60 (Urade, 2009; Liu and Howell, 2010).

Atbzip mutants do not display morphological or developmental differences under non-stress conditions, but are more sensitive to salt stress (Atbzip17, Liu et al., 2007b), heat (Atbzip28, Gao et al., 2008), or DTT treatment (Atbzip60, Humbert et al., 2012). The expression of salt stress responsive genes is impaired in Atbzip17 mutants (Liu et al., 2007b) as is the induction of canonical UPR genes in Atbzip28 and Atbzip60 mutants after TM treatment (Iwata and Koizumi, 2005a; Liu et al., 2007a; Iwata et al., 2008; Lu and Christopher, 2008; Tajima et al., 2008). Similar to ATF6 in mammals, AtbZIP17 and AtbZIP28 possess canonical S1P cleavage sites and are activated by a RIP-like process upon ER stress (Liu et al., 2007a,b, 2008a; Gao et al., 2008; Tajima et al., 2008; Che etal., 2010). RIP of AtbZIP17 and AtbZIP28 requires passage through the Golgi apparatus, where cleavage by the subtilisin-like serine protease AtS1P and subsequent processing by the metalloprotease AtS2P take place (Liu et al., 2007a,b; Che et al., 2010; Srivastava et al., 2012). How these bZIPs sense ER stress and how Golgi transition is mediated, is not clear. However, TM treatment apparently promotes the interaction of AtbZIP28 with the small GTPase SAR1b and the guanidine exchange factor SEC12, which are putatively involved in coat protein complex II (COPII) vesicle formation during ER-to-Golgi transport (Srivastava et al., 2012). AtbZIP60 lacks a canonical S1P cleavage site and its activation is independent of S1P and S2P (Iwata et al., 2008). Similar to mammalian XBP1 and yeast HAC1, recent studies in Arabidopsis and rice revealed unconventional splicing of a 23 nucleotide intron from the AtbZIP60 mRNA by AtIRE1b or AtIRE1a, and a 20 nucleotide intron from its rice orthologOsbZIP50/OsbZIP74 mRNA by OsIRE1, e.g., after TM or salicylic acid (SA) treatment. This leads to a frame shift that removes the transmembrane domain of the new proteins and allows nuclear entrance (Deng et al., 2011; Nagashima et al., 2011; Hayashi et al., 2012; Humbert et al., 2012; Lu et al., 2012; Moreno et al., 2012). There are no obvious PERK homologs in Arabidopsis (Koizumi et al., 2001; Urade, 2009). 


\section{ER STRESS AS INITIATOR OF PROGRAMED CELL DEATH}

The UPR is supposed to ensure cell survival. However, under prolonged or severe ER stress, mammalian cells activate an apoptosis-like programed cell death (ER-PCD) to eliminate damaged cells from stressed organisms (Schröder, 2006; Hetz, 2012; Jäger etal., 2012). The ER stress sensors ATF6, PERK, and IRE1 are central regulators of this process as well (Figure 1C), although it is unclear how they perceive and differentiate signals to switch from UPR to apoptosis. ER-PCD obviously merges with other apoptosis pathways, involving enhanced generation of reactive oxygen species (ROS), and apoptosis-promoting $\mathrm{Ca}^{2+}$ signaling at ER and mitochondria (Chakrabarti et al., 2011; Gorman etal., 2012; Hetz, 2012; Jäger et al., 2012). The induction of the pro-apoptotic bZIP transcription factor CHOP (C/EBPhomologs protein) by ATF6 and PERK/ATF4 during ER-PCD apparently is most relevant. CHOP down-regulates anti-apoptotic proteins (e.g., BCL-2), but induces members of the pro-apoptotic (BH3)-only protein family, e.g., BIM (BCL-2-INTERACTING MEDIATOR OF CELL DEATH) or GADD34 (GROWTH ARREST AND DNA DAMAGE-INDUCIBLE 34; Gorman et al., 2012; Hetz, 2012; Jäger et al., 2012). In addition, IRE1 activates ERPCD by interacting with TRAF2 (TUMOR NECROSIS FACTOR RECEPTOR-ASSOCIATED FACTOR 2; Gorman etal., 2012; Jäger et al., 2012). This initiates consecutive phosphorylation of ASK1 (APOPTOSIS SIGNAL-REGULATING KINASE 1) and JNK (JUN N-TERMINAL KINASE). Phosphorylation by JNK inactivates anti-apoptotic regulators such as BCL-2, but activates pro-apoptotic $\mathrm{BH} 3$-only proteins such as BIM or BID (BH3interacting domain death agonist). $\mathrm{BH} 3$-only proteins promote the cell death activation-related oligomerization and translocation of BAX and BAK to the mitochondrial membrane, followed by cytochrome $c$ release and caspase activation for execution of apoptosis. BCL-2-dependent regulation of $\mathrm{Ca}^{2+}$ homeostasis of the ER also affects permeability transition and apoptosis signaling at mitochondria (Chakrabarti et al., 2011; Gorman et al., 2012; Hetz, 2012). BAX and BAK themselves can interact with IRE1 and promote its ability to activate ASK1 and JNK, processes that are apparently blocked by the cell survival protein BI-1 (BAX INHIBITOR-1; Bailly-Maitre et al., 2009; Lisbona et al., 2009). Dynamic differential interactions with pro- and anti-apoptotic proteins modulated by the intensity and duration of ER stress signals might regulate separate functions of IRE1, and timely coordinated on- and offset of ATF6, PERK, and IRE1 signaling may play a decisive role in determining cell fate. In such a scenario, ER stress would initially activate the adaptive UPR via IRE1-mediated splicing of XBP1. However, down-regulation of the IRE1/XBP1 branch upon prolonged ER stress may give rise to pro-apoptotic IRE1/TREF2/ASK1/JNK, RIDD, and/or PERK signaling (Gorman et al., 2012; Hetz, 2012). Autophagy is further suggested to abolish ER stress in yeast and mammals as it might support the removal of unfolded proteins (Bernales et al., 2006). Here, the PERK-elF2 $\alpha$-ATF4 and IRE/TRAF2/JNK pathways might connect autophagy to ER stress via the BECLIN1BCL2 interaction and the induction of autophagy genes, respectively. Although ER stress-associated autophagy is thought to have a cytoprotective function, other studies suggest a role in ER-PCD. However, regulators of this cell death pathway and its link to ER stress are currently unknown (Verfaillie et al., 2010; Aronson and Davies, 2012).

As in animal cells, cell death follows induction of UPR in TMtreated plants (Zuppini et al., 2004; Iwata and Koizumi, 2005b; Watanabe and Lam, 2008; Ishikawa et al., 2011). The molecular basis of plant ER-PCD and the role of plant bZIPs therein are largely unknown (Figure 1D). However, regulation of ER-PCD seems to be partially conserved across kingdoms, as Arabidopsis BI-1 (AtBI-1) is involved in restriction of ER-PCD in Arabidopsis as well (Watanabe and Lam, 2008; Ishikawa et al., 2011). AtBI-1 is AtbZIP60-dependently up-regulated in response to TM (Kamauchi etal., 2005; Iwata etal., 2008; Watanabe and Lam, 2008). AtBI-1-mediated inhibition of ER-PCD in Arabidopsis is likely un-related to UPR modification, but rather to the suppression of ER-dependent ROS production or regulation of cell death associated $\mathrm{ER} \mathrm{Ca}^{2+}$ homeostasis (Watanabe and Lam, 2008, 2009). In Arabidopsis, a G $\beta$ subunit of an ER-resident heterotrimeric GTP-binding protein, AGB1, might be involved in the promotion of ER-PCD (Wang et al., 2007; Chen and Brandizzi, 2012). Disturbed ER protein retention after silencing of $N b E R D 2 a / N b E R D 2 b$ interferes with ER-QC and reduces ER stress alleviation, resulting in enhanced PCD in response to bacterial pathogens ( $\mathrm{Xu}$ et al., 2012). New insights into the role of vacuolar processing enzymes with caspasel-like activities in the execution of ER-PCD come from Qiang et al. (2012). These studies demonstrate the dependence of the mutualistic fungus Piriformospora indica on ER-PCD for successful Arabidopsis root colonization. P. indica induces ER stress but suppresses the adaptive UPR pathway. Consequently, the $P$. indica-induced ER stress triggers a vacuolar cell death pathway whose execution depends on $\gamma$ VACUOLAR PROCESSING ENZYME ( $\gamma$ VPE). This ER-PCD can be phenocopied by the application of TM to Arabidopsis roots. The analyses further show that $\gamma \mathrm{VPE}$ is responsible for enhanced VPE and caspase 1-like activities during TM- and P. indica-induced ER-PCD (Qiang et al., 2012).

\section{ER - EXECUTOR OF PLANT IMMUNITY AND PUTATIVE TARGET OF PATHOGEN EFFECTORS}

Plants ward off pathogens by a multi-layered immune system. PM localized pattern recognition receptors (PRRs) detect conserved molecules, so-called microbe-associated molecular patterns (MAMPs), of invading microbes. Well-characterized PRRs are FLAGELLIN-SENSING 2 (FLS2), which recognizes bacterial flagellin, the ELONGATION-FACTOR TU (EF-Tu) RECEPTOR (EFR), which detects bacterial EF-Tu, and the chitin receptors CHITIN ELICITOR BINDING PROTEIN (CEBiP) and CHITIN ELICITOR RECEPTOR KINASE (CERK; Monaghan and Zipfel, 2012). MAMP perception by these PRRs initiates immune signaling pathways, defined as MAMP-triggered immunity (MTI), which involve $\mathrm{Ca}^{2+}$ fluxes across the $\mathrm{PM}$, a rapid production of ROS, the activation of mitogen-activated protein kinase cascades and WRKY transcription factors, eventually resulting in the induction of defense mechanisms including callose depositions and the synthesis of antimicrobial pathogenesis-related (PR) proteins (Jones and Dangl, 2006; Boller and Felix, 2009). Successful pathogens have evolved effector molecules to suppress MTI. Plant RESISTANCE (R) proteins specifically recognize pathogen effectors or their activities and initiate effector-triggered immunity 
(ETI), typically involving hypersensitive response (HR)-related PCD (Chisholm etal., 2006; Jones and Dangl, 2006). The ER participates in plant innate immunity in several ways. Firstly, immunity depends on the secretory apparatus for the production of immune proteins (Wang et al., 2005; Nekrasov et al., 2009; Saijo et al., 2009). NONEXPRESSOR OF PR GENES 1 (NPR1), the master regulator of SA-dependent systemic acquired resistance (SAR), coordinately controls the up-regulation of $P R$ genes and genes encoding proteins of the secretory pathway during SAR (Wang et al., 2005). Secondly, synthesis and proper function of PRRs (e.g., EFR) rely on $N$-glycosylation and the ER-QC system, which involves staurosporine and temperature sensitive-3a (STT3A), glucosidase II, the H/KDEL receptor ERD2b, the UDPglucose:glycoprotein glucosyltransferase (UGGT)/CRT3 cycle and the stromal cell-derived factor-2 (SDF2)/ERdj3B/BiP complex (Li et al., 2009; Lu et al., 2009; Nekrasov et al., 2009; Saijo et al., 2009; Saijo, 2010). Susceptibility of ER-QC mutants to pathogens differs qualitatively and quantitatively from that of efr mutants, suggesting the existence of EFR-independent but ER-QC-dependent immune response (Li et al., 2009; Nekrasov et al., 2009; Saijo et al., 2009). Meanwhile, a number of membrane-localized immune receptors have been identified, whose functions depend on ERQC, among them the rice PRR XA21 involved in resistance to Xanthomonas oryzae pv. oryzae (Park et al., 2010a,b), an induced receptor kinase (IRK), which is involved in $\mathrm{N}$-mediated resistance of tobacco to tobacco mosaic virus (Caplan et al., 2009), and glycosylated Cf proteins, which confer race-specific resistance to the fungal pathogen Cladosporium fulvum (Liebrand et al., 2012). Similar to FLS2, the ER-QC disturbance does not affect CERK1 function in Arabidopsis (Li et al., 2009; Nekrasov et al., 2009). However, the rice homolog OsCERK1 seems to interact with a Hop/Stil-Hsp90 chaperone complex for maturation in the ER prior to transport to the PM (Chen et al., 2010). ER-QC also monitors glycosylation and proper folding of some immunity-related Toll-like receptors (TLRs) that recognize MAMPs in animals (Yang et al., 2007). Interestingly, PRRs TLR4 and TLR2 activate the IRE1 $\alpha$-XBP1 pathway to enhance secretion of certain proinflammatory cytokines in macrophages, and loss of XBP1 function impairs immunity against the bacterial pathogen Francisella tularensis (Martinon et al., 2010).

Induction of the ER-QC machinery accompanies synthesis of immunity-associated proteins in plants (Jelitto-Van Dooren et al., 1999; Wang et al., 2005). Consequently, ER-QC mutants are more susceptible to ER stress inducers and pathogens (Wang et al., 2005; Li et al., 2009; Lu et al., 2009; Nekrasov et al., 2009; Saijo et al., 2009). Similarly, proper execution of defense responses may rely on the induction of UPR genes. Recently, the heat-shock factor-like transcription factor TBF1 has been identified as important transcriptional regulator of UPR genes, and Arabidopsis tbf 1 mutants are impaired in the execution of SAR and EFR-mediated MTI (Pajerowska-Mukhtar et al., 2012). The Nicotiana benthamiana homolog of AtbZIP60, NbbZIP60, is induced in response to

\section{REFERENCES}

Aronson, L. I., and Davies, F. E.

(2012). DangER: protein ovERload

- targeting protein degradation to treat myeloma. Haematologica 97, 1119-1130.

Bailly-Maitre, B., Belgardt, B. F., Jordan, S. D., Coornaert, B., von Freyend, M.

inoculation with avirulent Pseudomonas cichorii and required to arrest its growth (Tateda et al., 2008). Furthermore, AtIRE1a and $A t I R E 1 b$ expression is pathogen-responsive, and both proteins are required for SA or pathogen-dependent splicing of AtbZIP60, expression of ER-QC genes, secretion of defense proteins and thus execution of SAR (Moreno et al., 2012).

Together, this underlines the functional importance of the ER in both MTI and ETI, and designates it as a potential effector target. Consistent with this, many viruses employ host UPR by targeting ER stress sensors to enhance folding of viral proteins or to modulate immune responses in mammals (Ke and Chen, 2011; Qian et al., 2012). In tobacco, infection with Potato virus X or overexpression of a viral movement protein induces bZIP60 and UPR genes possibly to suppress host cell death responses (Ye et al., 2011). In addition, Yamamoto et al. (2011) showed that ATF6 $\beta$ is part of mice immunity against the protozoan parasite Toxoplasma gondii. ROP18, a serine/threonine kinase, which is secreted into the host cell during infection, interacts with ATF6 $\beta$ and mediates its proteasome-dependent degradation. Thus, ATF6 $\beta$ constitutes a target for the T. gondii ROP18 virulence factor possibly to suppress UPR-mediated host defense. Likewise, the Salmonella enterica leucine-rich repeat (LRR) effector protein SlrP targets the host ER-QC member ERdj3. This supports infection as it leads to the accumulation of unfolded proteins eventually promoting host cell death (Bernal-Bayard et al., 2010). In Caenorhabditis elegans, the increased requirement of secreted proteins during the activation of immune responses imposes ER stress to the organism itself, which requires XBP1mediated UPR to avoid onset of ER-PCD (Richardson et al., 2010). Several bacterial toxins, e.g., Shiga toxin produced by enterohemorrhagic bacteria, can enter the ER and seem to initiate cell death through prolonged UPR signaling by activating ER stress sensors (Tesh, 2012).

\section{CONCLUSIONS AND PERSPECTIVE}

As production site of antimicrobial proteins and of immune signaling components, the ER functions as central regulator in the execution of immune responses in plants and animals. Therefore, the disturbance of ER integrity is certainly of primary relevance for pathogens to achieve host cell infection. Plants further rely on proper ER function and likely ER membrane localized stress sensors for adaptation to abiotic stress such as salt or heat stress (Liu et al., 2008a,b, 2011; Che et al., 2010; Cui et al., 2012). Taken together, the improvement of plant UPR in order to maintain ER homeostasis under unfavorable conditions may increase plant adaptability to biotic and abiotic stress, which bears a potential to enhance crop yield and yield stability.

\section{ACKNOWLEDGMENTS}

We kindly thank the German Research Council (Deutsche Forschungsgemeinschaft) for financial support through grants to Ruth Eichmann (EI835/1-1) and Patrick Schäfer (SCHA1444/3-3).

J., Kleinridders, A., Mauer, J., Cuddy, M., Kress, C. L., Willmes, D., Essig, M., Hampel, B., Protzer, U., Reed, J. C., and Brüning, J. C. (2009). Hepatic
Bax inhibitor-1 inhibits IRE1 $\alpha$ and protects from obesity-associated insulin resistance and glucose intolerance. J. Biol. Chem. 285, 6198-6207. 
Bernal-Bayard, J., Cardenal-Muñoz, E., and Ramos-Morales, F. (2010). The Salmonella type III secretion effector, Salmonella leucine-rich repeat protein (SlrP), targets the human chaperone ERdj3. J. Biol. Chem. 285, 1636016368.

Bernales, S., McDonald, K. L., and Walter, P. (2006). Autophagy counterbalances endoplasmic reticulum expansion during the unfolded protein response. PLoS Biol. 4, e423. doi: 10.1371/journal.pbio.0040423

Boller, T., and Felix, G. (2009). A renaissance of elicitors: perception of microbe-associated molecular patterns and danger signals by patternrecognition receptors. Annu. Rev. Plant Biol. 60, 379-406.

Caplan, J. L., Zhu, X., Mamillapalli, P., Marathe, R., Anandalakshmi, R., and Dinesh-Kumar, S. P. (2009). Induced ER chaperones regulate a receptorlike kinase to mediate antiviral innate immune response in plants. Cell Host Microbe 6, 457-469.

Chakrabarti, A., Chen, A. W., and Varner, J. D. (2011). A review of the mammalian unfolded protein response. Biotechnol. Bioeng. 108, 2777-2793.

Che, P., Bussell, J. D., Zhou, W. Estavillo, G. M., Pogson, B. J., and Smith, S. M. (2010). Signaling from the endoplasmic reticulum activates brassinosteroid signaling and promotes acclimation to stress in Arabidopsis. Sci. Signal. 3, ra69.

Chen, L., Hamada, S., Fujiwara, M., Zhu, T., Thao, N. P., Wong, H. L., Krishna, P., Ueda, T., Kaku, H., Shibuya, N., Kawasaki, T., and Shimamoto, K. (2010). The Hop/Stil-Hsp90 chaperone complex facilitates the maturation and transport of a PAMP receptor in rice innate immunity. Cell Host Microbe 7 185-196.

Chen, Y., and Brandizzi, F. (2012). AtIRE1A/AtIRE1B and AGB1 independently control two essential unfolded protein response pathways in Arabidopsis. Plant J. 69, 266-277.

Chisholm, S. T., Coaker, G., Day, B., and Staskawicz, B. J. (2006). Host-microbe interactions: shaping the evolution of the plant immune response. Cell 124, 803-814.

Cui, F., Liu, L., Zhao, Q., Zhang, Z., Li, Q., Lin, B., Wu, Y., Tang, S., and Xie, Q. (2012). Arabidopsis ubiquitin conjugase UBC32 is an ERAD component that functions in brassinosteroid-mediated salt stress tolerance. Plant Cell 24, 233-244.

Deng, Y., Humbert, S., Liu, J. X., Srivastava, R., Rothstein, S. J., and Howell,
S. H. (2011). Heat induces the splicing by IRE1 of a mRNA encoding a transcription factor involved in the unfolded protein response in Arabidopsis. Proc. Natl. Acad. Sci. U.S.A. $108,7247-7252$.

Gao, H., Brandizzi, F., Benning, C., and Larkin, R. M. (2008). A membranetethered transcription factor defines a branch of the heat stress response in Arabidopsis thaliana. Proc. Natl. Acad. Sci. U.S.A. 105, 16398-16403.

Gorman, A. M., Healy, S. J., Jäger, R., and Samali, A. (2012). Stress management at the ER: regulators of ER stress-induced apoptosis. Pharmacol. Ther. 134, 306-316.

Harding, H. P., Novoa, I., Zhang Y., Zeng, H., Wek, R., Schapira, M., and Ron, D. (2000). Regulated translation initiation controls stressinduced gene expression in mammalian cells. Mol. Cell 6, 10991108.

Hayashi, S., Wakasa, Y., Takahashi, H., Kawakatsu, T., and Takaiwa, F. (2012). Signal transduction by IRE1mediated splicing of bZIP50 and other stress sensors in the endoplasmic reticulum stress response of rice. Plant J. 69, 946-956.

Hetz, C. (2012). The unfolded protein response: controlling cell fate decisions under ER stress and beyond. Nat. Rev. Mol. Cell Biol. 13, 89-102.

Higa, A., and Chevet, E. (2012). Redox signaling loops in the unfolded protein response. Cell. Signal. 24, 15481555.

Humbert, S., Zhong, S., Deng, Y., Howell, S. H., and Rothstein, S. J. (2012). Alteration of the bZIP60/IRE1 pathway affects plant response to ER stress in Arabidopsis thaliana. PLoS ONE 7, e39023. doi: 10.1371/journal.pone. 0039023

Hüttner, S., and Strasser, R. (2012). Endoplasmic reticulum-associated degradation of glycoproteins in plants. Front. Plant Sci. 3: 67. doi: 10.3389/fpls.2012.00067

Ishikawa, T., Watanabe, N., Nagano, M., Kawai-Yamada, M., and Lam, E. (2011). Bax inhibitor-1: a highly conserved endoplasmic reticulum-resident cell death suppressor. Cell Death Differ. 18, 1271-1278.

Iwata, Y., Fedoroff, N. V., and Koizumi, N. (2008). Arabidopsis bZIP60 is a proteolysis-activated transcription factor involved in the endoplasmic reticulum stress response. Plant Cell 20,3107-3121

Iwata, Y., and Koizumi, N. (2005a). An Arabidopsis transcription factor AtbZIP60, regulates the endoplasmic reticulum stress response in a manner unique to plants. Proc. Natl. Acad. Sci. U.S.A. 102, 5280-5285.

Iwata, Y., and Koizumi, N. (2005b). Unfolded protein response followed by induction of cell death in cultured tobacco cells treated with tunicamycin. Planta 220, 804-807.

Jäger, R., Bertrand, M. J., Gorman, A. M., Vandenabeele, P., and Samali, A. (2012). The unfolded protein response at the crossroads of cellular life and death during endoplasmic reticulum stress. Biol. Cell 104, 259-270.

Jelitto-Van Dooren, E. P., Vidal, S., and Denecke, J. (1999). Anticipating endoplasmic reticulum stress. A novel early response before pathogenesis-related gene induction Plant Cell 11, 1935-1944.

Jones, J. D., and Dangl, J. L. (2006). The plant immune system. Nature 444, 323-329.

Kamauchi, S., Nakatani, H., Nakano, C., and Urade, R. (2005). Gene expression in response to endoplasmic reticulum stress in Arabidopsis thaliana. FEBS J. 272, 3461-3476.

Ke, P. Y., and Chen, S. S. (2011). Activation of the unfolded protein response and autophagy after hepatitis $C$ virus infection suppresses innate antivira immunity in vitro. J. Clin. Invest. 121 , 37-56.

Koizumi, N., Martinez, I. M., Kimata, Y., Kohno, K., Sano, H., and Chrispeels, M. J. (2001) Molecular characterization of two Arabidopsis Irel homologs, endoplasmic reticulum-located transmembrane protein kinases. Plant Physiol. 127, 949-962.

Leborgne-Castel, N., Jelitto-Van Dooren, E. P. W. M., Crofts, A J., and Denecke, J. (1999). Overexpression of $\mathrm{BiP}$ in tobacco alleviates endoplasmic reticulum stress. Plant Cell 11, 459-469.

Li, J., Zhao-Hui, C., Batoux, M., Nekrasov, V., Roux, M., Chinchilla, D., Zipfel, C., and Jones, J. D. (2009). Specific ER quality control components required for biogenesis of the plant innate immune receptor EFR. Proc. Natl. Acad. Sci. U.S.A. 106, 15973-15978.

Liebrand, T. W., Smit, P., Abd-ElHaliem, A., de Jonge, R., Cordewener, J. H., America, A. H., Sklenar, J., Jones, A. M., Robatzek, S., Thomma, B. P., Tameling, W. I., and Joosten M. H. (2012). ER-quality control chaperones facilitate the biogenesis of Cf receptor-like proteins involved in pathogen resistance of tomato. Plant Physiol. 159, 1819-1833.

Lisbona, F., Rojas-Rivera, D., Thielen, P. Zamorano, S., Todd, D., Martinon, F.,
Glavic, A., Kress, C., Lin, J. H., Walter, P., Reed, J. C., Glimcher, L. H., and Hetz, C. (2009). BAX inhibitor-1 is a negative regulator of the ER stress sensor IRE1 $\alpha$. Mol. Cell 33, 679-691.

Liu, J. X., and Howell, S. H. (2010). Endoplasmic reticulum protein quality control and its relationship to environmental stress responses in plants. Plant Cell 22, 2930-2942.

Liu, J. X., Srivastava, R., Che, P., and Howell, S. H. (2007a). An endoplasmic reticulum stress response in Arabidopsis is mediated by proteolytic processing and nuclear relocation of a membrane-associated transcription factor, bZIP28. Plant Cell 19, 4111-4119.

Liu, J. X., Srivastava, R., Che, P., and Howell, S. H. (2007b). Salt stress responses in Arabidopsis utilize a signal transduction pathway related to endoplasmic reticulum stress signaling. Plant J. 51, 897-909.

Liu, J. X., Srivastava, R., Che, P., and Howell, S. H. (2008a). Salt stress signaling in Arabidopsis thaliana involves a membrane-bound transcription factor AtbZIP17 as a signal transducer. Plant Signal. Behav.3, 56-57.

Liu, J. X., Srivastava, R., and Howell, S. H. (2008b). Stress-induced expression of an activated form of AtbZIP17 provides protection from salt stress in Arabidopsis. Plant Cell Environ. 31, 1735-1743.

Liu, L., Cui, F., Li, Q., Yin, B., Zhang, H., Lin, B., Wu, Y., Xia, R., Tang, S., and Xie, Q. (2011). The endoplasmic reticulum-associated degradation is necessary for plant salt tolerance. Cell Res. 21, 957-969.

Lu, D. P., and Christopher, D. A. (2008). Endoplasmic reticulum stress activates the expression of a subgroup of protein disulfideisomerase genes and AtbZIP60 modulates the response in Arabidopsis thaliana. Mol. Genet. Genomics 280, 199-210.

Lu, S. J., Yang, Z. T., Sun, L., Sun, L., Song, Z. T., and Liu, J. X. (2012). Conservation of IRE1-regulated bZIP74 mRNA unconventional splicing in rice (Oryza sativa L.) involved in ER stress responses. Mol. Plant 5, 504-514.

Lu, X., Tintor, N., Mentzel, T., Kombrink, E., Boller, T., Robatzek, S., Schulze-Lefert, P., and Saijo, Y. (2009). Uncoupling of sustained MAMP receptor signaling from early outputs in an Arabidopsis endoplasmic reticulum glucosidase II allele. Proc. Natl. Acad. Sci. U.S.A. 106, 22522-22527.

Lynes, E. M., and Simmen, T. (2011). Urban planning of the endoplasmic 
reticulum (ER): how diverse mechanisms segregate the many functions of the ER. Biochim. Biophys. Acta 1813, 1893-1905.

Martínez, I. M., and Chrispeels, M. J. (2003). Genomic analysis of the unfolded protein response in Arabidopsis shows its connection to important cellular processes. Plant Cell 15, 561-576.

Martinon, F., Chen, X., Lee, A. H., and Glimcher, L. H. (2010). TLR activation of the transcription factor XBP1 regulates innate immune responses in macrophages. Nat. Immunol. 11, 411-418.

Monaghan, J., and Zipfel, C. (2012). Plant pattern recognition receptor complexes at the plasma membrane. Curr. Opin. Plant Biol. 15, 349-357.

Moreno, A. A., Mukhtar, M. S., Blanco, F., Boatwright, J. L., Moreno, I. Jordan, M. R., Chen, Y., Brandizzi, F., Dong, X., Orellana, A., and Pajerowska-Mukhtar, K. M. (2012). IRE1/bZIP60-mediated unfolded protein response plays distinct roles in plant immunity and abiotic stress responses. PLoS ONE 7, e31944. doi: 10.1371/journal.pone. 0031944

Mori, K. (2009). Signalling pathways in the unfolded protein response: development from yeast to mammals. $J$ Biochem. 146, 743-750.

Nagashima, Y., Mishiba, K., Suzuki, E., Shimada, Y., Iwata, Y., and Koizumi, N. (2011). Arabidopsis IRE1 catalyses unconventional splicing of bZIP60 mRNA to produce the active transcription factor. Sci. Rep. 1, 29.

Nekrasov, V., Li, J., Batoux, M., Roux, M., Chu, Z. H., Lacombe, S., Rougon, A., Bittel, P., Kiss-Papp, M., Chinchilla, D., van Esse, H. P., Jorda, L., Schwessinger, B., Nicaise, V., Thomma, B. P., Molina, A., Jones, J. D., and Zipfel, C. (2009). Control of the pattern-recognition receptor EFR by an ER protein complex in plant immunity. EMBO J. 28, 3428-3438.

Noh, S. J., Kwon, C. S., and Chung, W. I. (2002). Characterization of two homologs of Irelp, a kinase/endoribonuclease in yeast, in Arabidopsis thaliana. Biochim. Biophys. Acta 1575, 130-134.

Okushima, Y., Koizumi, N., Yamaguchi, Y., Kimata, Y., Kohno, K., and Sano, H. (2002). Isolation and characterization of a putative transducer of endoplasmic reticulum stress in Oryza sativa. Plant Cell Physiol. 43, 532-539.

Pajerowska-Mukhtar, K. M., Wang, W., Tada, Y., Oka, N., Tucker, C. L., Fonseca, J. P., and Dong, X. (2012). The HSF-like transcription factor TBF1 is a major molecular switch for plant growth-to-defense transition. Curr. Biol. 22, 103-112.

Park, C. J., Bart, R., Chern, M., Canlas, P. E., Bai, W., and Ronald, P. C. (2010a). Overexpression of the endoplasmic reticulum chaperone $\mathrm{BiP} 3$ regulates XA21-mediated innate immunity in rice. PLoS ONE 5, e9262. doi: 10.1371/journal.pone.0009262

Park, C. J., Han, S. W., Chen, X., and Ronald, P. C. (2010b). Elucidation of XA21-mediated innate immunity. Cell. Microbiol. 12, 1017-1025.

Qian, Z., Xuan, B., Chapa, T. J., Gualberto, N., and Yu, D. (2012). Murine cytomegalovirus targets transcription factor ATF4 to exploit the unfolded-protein response. J. Virol. $86,6712-6723$.

Qiang, X., Zechmann, B., Reitz, M. U., Kogel, K. H., and Schäfer, P. (2012). The mutualistic fungus Piriformospora indica colonizes Arabidopsis roots by inducing an endoplasmic reticulum stress-triggered caspasedependent cell death. Plant Cell 24 794-809.

Richardson, C. E., T. Kooistra, and Kim, D. H. (2010). An essential role for XBP-1 in host protection against immune activation in $C$. elegans. Nature 463, 1092-1095.

Saijo, Y. (2010). ER quality control of immune receptors and regulators in plants. Cell. Microbiol. 12, 716-724.

Saijo, Y., Tintor, N., Lu, X., Rauf, P., Pajerowska-Mukhtar, K., Häweker, H., Dong, X., Robatzek, S., and Schulze-Lefert, P. (2009). Receptor quality control in the endoplasmic reticulum for plant innate immunity. EMBO J. 28, 3439-3449.

Schröder, M. (2006). The unfolded protein response. Mol. Biotechnol. 34, 279-290.

Schröder, M. (2008). Endoplasmic reticulum stress responses. Cell. Mol. Life. Sci. 65, 862-894.

Sparkes, I. A., Frigerio, L., Tolley, N., and Hawes, C. (2009). The plant endoplasmic reticulum: a cell-wide web. Biochem. J. 423,145-155.

Srivastava, R., Chen, Y., Deng, Y., Brandizzi, F., and Howell, S. H. (2012). Elements proximal to and within the transmembrane domain mediate the organelle-to-organelle movement of bZIP28 under ER stress conditions. Plant J. 70, 1033-1042.

Staehelin, L. A. (1997). The plant ER: a dynamic organelle composed of a large number of discrete functional domains. Plant J. 11, 1151-1165.

Su, W., Liu, Y., Xia, Y., Hong, Z., and Li, J. (2011). Conserved endoplasmic reticulum-associated degradation system to eliminate mutated receptor-like kinases in Arabidopsis.
Proc. Natl. Acad. Sci. U.S.A. 108 870-875.

Tajima, H., Iwata, Y., Iwano, M. Takayama, S., and Koizumi, N. (2008). Identification of an Arabidopsis transmembrane bZIP transcription factor involved in the endoplasmic reticulum stress response. Biochem. Biophys. Res. Commun. 374, 242-247.

Tateda, C., Ozaki, R., Onodera, Y., Takahashi, Y., Yamaguchi, K., Berberich, T., Koizumi, N., and Kusano, T. (2008). NtbZIP60, an endoplasmic reticulum-localized transcription factor, plays a role in the defense response against bacterial pathogen in Nicotiana tabacum. I. Plant Res. 121, 603-611.

Tesh, V. L. (2012). Activation of cell stress response pathways by Shiga toxins. Cell. Microbiol. 14, 1-9.

Urade, R. (2009). The endoplasmic reticulum stress signaling pathways in plants. Biofactors 35, 326-331.

Vitale, A., and Boston, R. S. (2008). Endoplasmic reticulum quality control and the unfolded protein response: insights from plants. Traffic 9, 1581-1588.

Verfaillie, T., Salazar, M., Velasco, G., and Agostinis, P. (2010). Linking ER stress to autophagy: potential implications for cancer therapy. Int. J. Cell Biol. 2010, 930509.

Walter, P., and Ron, D. (2011). The unfolded protein response: from stress pathway to homeostatic regulation. Science 334, 1081-1086.

Wang, D., Weaver, N. D., Kesarwani, M. and Dong, X. (2005). Induction of protein secretory pathway is required for systemic acquired resistance. Science 308, 1036-1040.

Wang, S., Narendra, S., and Fedoroff, N. (2007). Heterotrimeric G protein signaling in the Arabidopsis unfolded protein response. Proc. Natl. Acad. Sci. U.S.A. 104, 3817-3822.

Watanabe, N., and Lam, E. (2008). BAX inhibitor-1 modulates endoplasmic reticulum stress-mediated programmed cell death in Arabidopsis. J. Biol. Chem. 283, 32003210.

Watanabe, N., and Lam, E. (2009). Bax inhibitor-1, a conserved cell death suppressor, is a key molecular switch downstream from a variety of biotic and abiotic stress signals in plants. Int. J. Mol. Sci. 10, 31493167.

Xu, G., Li, S., Xie, K., Zhang, Q., Wang, Y., Tang, Y., Liu, D. Hong, Y., He, C., and Liu, Y. (2012). Plant ERD2-like proteins function as ER luminal protein receptors and participate in programmed cell death during innate immunity. Plant J. doi: 10.1111/j.1365313X.2012.05053.x [Epub ahead of print].

Yamamoto, M., Ma, J. S., Mueller, C., Kamiyama, N., Saiga, H., Kubo, E., Kimura, T., Okamoto, T., Okuyama, M., Kayama, H., Nagamune, K., Takashima, S., Matsuura, Y., SoldatiFavre, D., and Takeda, K. (2011). ATF6 $\beta$ is a host cellular target of the Toxoplasma gondii virulence factor ROP18. J. Exp. Med. 208, 1533-1546.

Yang, Y., Liu, B., Dai, J., Srivastava, P. K., Zammit, D. J., Lefrancois, L., and Li, Z. (2007). Heat shock protein gp96 is a master chaperone for toll-like receptors and is important in the innate function of macrophages. Immunity 26, 215-226.

Ye, C., Dickman, M. B., Whitham, S. A., Payton, M., and Verchot, J. (2011). The unfolded protein response is triggered by a plant viral movement protein. Plant Physiol. 156, 741-755.

Yoshida, H., Matsui, T., Yamamoto, A., Okada, T., and Mori, K. (2001). XBP1 mRNA is induced by ATF6 and spliced by IRE1 in response to ER stress to produce a highly active transcription factor. Cell 107, 881-891.

Zuppini, A., Navazio, L., and Mariani, P. (2004). Endoplasmic reticulum stress-induced programmed cell death in soybean cells. J. Cell Sci. 117, 2591-2598.

Conflict of Interest Statement: The authors declare that the research was conducted in the absence of any commercial or financial relationships that could be construed as a potential conflict of interest.

Received: 02 July 2012; paper pending published: 16 July 2012; accepted: 09 August 2012; published online: 22 August 2012.

Citation: Eichmann $R$ and Schäfer $P$ (2012) The endoplasmic reticulum in plant immunity and cell death. Front. Plant Sci. 3:200. doi: 10.3389/fpls.2012. 00200

This article was submitted to Frontiers in Plant Proteomics, a specialty of Frontiers in Plant Science.

Copyright $\odot 2012$ Eichmann and Schäfer. This is an open-access article distributed under the terms of the Creative Commons Attribution License, which permits use, distribution and reproduction in other forums, provided the original authors and source are credited and subject to any copyright notices concerning any third-party graphics etc. 